\title{
Cuidados em emergência: conhecimento e aplicabilidade do protocolo de Manchester por enfermeiros
}

\author{
Emergency care: knowledge and applicability of the Manchester protocol by nurses \\ Atención de emergencia: conocimiento y aplicabilidad del protocolo de Manchester por enfermeras
}

Recebido: 11/10/2021 | Revisado: 16/10/2021 | Aceito: 22/10/2021 | Publicado: 24/10/2021

\author{
Davyd da Conceição Lima \\ ORCID: https://orcid.org/0000-0003-4194-4561 \\ Centro Universitário Santo Agostinho, Brasil \\ E-mail: davydlimac88@gmail.com \\ George Robson Ibiapina II \\ ORCID: https://orcid.org/0000-0001-7952-164X \\ Centro Universitário Santo Agostinho, Brasil \\ E-mail: georgeribiapina@gmail.com \\ Államy Danilo Moura e Silva \\ ORCID: https://orcid.org/0000-0001-7368-5395 \\ Centro Universitário Santo Agostinho, Brasil \\ E-mail: allamydanilo@hotmail.com
}

\begin{abstract}
Resumo
Trata-se de uma revisão integrativa de literatura acerca da triagem de urgência e aplicabilidade do protocolo de Manchester por enfermeiros, profissional que vem sendo responsável por essa demanda. O principal objetivo do estudo foi evidenciar quais os conhecimentos necessários para aplicação do protocolo de Manchester por enfermeiros. A pesquisa ocorreu nas bases de dados "LILACS", "BDENF" e "MEDLINE" via BVS. Concluiu-se que há a necessidade de mais estudos sobre o assunto, além de elencar os conhecimentos necessários para aplicação desse protocolo pelo enfermeiro.
\end{abstract}

Palavras-chave: Enfermeiro; Enfermagem em emergência; Cartão de triagem.

\begin{abstract}
This is an integrative literature review on emergency screening and applicability of the Manchester protocol by nurses, professional who has been responsible for this demand. The main objective of the study was to show what knowledge is needed for the application of the Manchester protocol by nurses. The search took place in the "LILACS", "BDENF" and "MEDLINE" databases via the BVS. It was concluded that there is a need for more studies on the subject, in addition to listing the knowledge necessary for the application of this protocol by the nurse.
\end{abstract}

Keywords: Nurse; Emergency nursing; Screening card.

\section{Resumen}

Se trata de una revisión integradora de la literatura sobre el cribado de emergencia y la aplicabilidad del protocolo de Manchester por parte de las enfermeras, profesional que ha sido responsable de esta demanda. El objetivo principal del estudio fue mostrar qué conocimientos se necesitan para la aplicación del protocolo de Manchester por parte de las enfermeras. La búsqueda se realizó en las bases de datos "LILACS", "BDENF" y "MEDLINE" a través de la BVS. Se concluyó que existe la necesidad de realizar más estudios sobre el tema, además de enumerar los conocimientos necesarios para la aplicación de este protocolo por parte de las enfermeras.

Palabras clave: Enfermero; Enfermería de emergencia; Tarjeta de cribado.

\section{Introdução}

A triagem é a porta de entrada para atendimento de urgência nos serviços hospitalares e deve ser realizada de maneira rápida e precisa por profissionais capacitados e habilitados. $\mathrm{O}$ atendimento de urgência tem como principal característica a necessidade de rapidez no atendimento sem a perda da qualidade. Existem modelos de classificação de risco que se diferem quanto a quantidade de níveis, sendo o de 5 níveis o mais adequado. O Protocolo de Manchester, protocolo mais utilizado no Brasil, foi criado com o objetivo de organizar o atendimento nos serviços de urgências e assegurar que pacientes não esperem mais do que o tempo necessário para o primeiro atendimento médico (Aguiar, 2019). 
Esse protocolo organiza os pacientes por cores após uma avaliação que consiste na observação de alguns parâmetros como sinais vitais, sintomas e nível de dor, que representam a gravidade do quadro e o tempo de espera para o atendimento de cada cliente. A Classificação de Risco deve ser realizada por um profissional de nível superior devidamente preparado. O enfermeiro vem sendo a figura principal na tarefa de triar os pacientes que buscam o serviço de urgência. A classificação de risco é uma ferramenta que auxilia o enfermeiro a assumir a função na regulação da demanda assistencial e na determinação da prioridade no atendimento desses clientes (Roncalli et al., 2017).

No âmbito da área de urgência, principalmente em relação ao protocolo de Manchester, o critério de acesso ao serviço é a gravidade. Os pacientes em situação de urgência serão atendidos em primeiro lugar. Porém, muitas vezes, pacientes em situações não urgentes também procuram esse tipo de serviço por ser, na teoria, mais acessível. Isto cria uma série de problemas para o profissional responsável pela triagem, o que resulta na superlotação e na sobrecarga da equipe que atua na área (Soares Acl, 2018).

$\mathrm{O}$ atendimento na triagem de urgência é a base para o decorrer dos procedimentos e o protocolo de Manchester é a maneira de organizar a prioridade desse atendimento, que é realizado pelo enfermeiro. Tendo isso em vista, é de suma importância identificar e analisar quais os conhecimentos necessários para a aplicação desse procedimento, revisando as evidencia cientificas presentes na literatura. O objetivo desse estudo consiste em revisar na literatura quais os conhecimentos necessários para a aplicabilidade do protocolo de Manchester por enfermeiros.

\section{Metodologia}

Quadro 1: Descritores controlados e não controlados utilizados para busca nas bases de dados, Teresina, PI, 2021.

\begin{tabular}{|l|l|l|}
\hline DeCS & Descritores controlados & Descritores não controlados \\
\hline P & Enfermeiro & Papel da enfermagem \\
\hline I & Enfermagem em Emergência & Classificação \\
\hline Co & Cartão de triagem & Classificação de Manchester. \\
\hline
\end{tabular}

Fonte: Autores.

Trata-se de uma revisão integrativa de literatura. Este método permite a busca, a síntese e a avaliação das informações disponíveis sobre um tema investigado, resumindo o passado da literatura, para fornecer uma análise mais abrangente de um determinado tema. A estrutura do estudo se dividiu em 5 etapas: escolha do tema, elaboração do problema de pesquisa, delimitação das informações a serem extraídas dos artigos, analise e interpretação das amostras e elaboração da síntese dos resultados (Mendes et al., 2019).

Foram adotados os seguintes critérios de inclusão: estudos que abordarem a enfermagem de emergência, classificação e triagem de urgência baseado no protocolo de Manchester e os conhecimentos necessários para sua aplicação nos idiomas português, espanhol e inglês. Serão excluídos todos os estudos que não abordarem o assunto proposto. Os artigos duplicados foram contabilizados apenas uma vez.

A pesquisa ocorreu nas seguintes bases de dados: LILACS (Literatura Latino-Americana e do Caribe em Ciências da Saúde,); Medical Literature Analysis and Retrieval System Online (MEDLINE); Base de Dados em Enfermagem (BDENF)via Biblioteca Virtual em Saúde (BVS). A questão de pesquisa foi estruturada de acordo com a estratégia PICo (PEnfermeiros/papel do enfermeiro; I- enfermagem de emergência/classificação; Co- classificação de Manchester/cartão de triagem. Com base nessa estratégia, elaborou-se a seguinte questão: Quais as evidências científicas disponíveis sobre o conhecimento e a aplicabilidade do protocolo de Manchester por enfermeiros? 
Foram utilizados os descritores controlados do Banco de Descritores em Ciências da Saúde (DeCS) Medical Subject Headings $(\mathrm{MeSH})$. Para ampliar a pesquisa, foram utilizadas palavras chaves e sinônimos em inglês e português. Foi empregado, para sistematização da amostra, o sistema de operadores booleanos “OR” e "AND”.

A busca foi realizada por dois pesquisadores independentes, de forma simultânea, em que padronizaram a sequência de utilização dos descritores e dos cruzamentos em cada base de dados e, em seguida, compararam os resultados obtidos. Todos os estudos foram acessados por meio do portal de periódicos da Coordenação de Aperfeiçoamento de Pessoal de Nível Superior (CAPES), com Internet Protocol (IP) autenticada na Universidade Federal do Piauí, visando garantir a busca ampla.

O Quadro 2 apresenta a estratégia de busca realizada na base de dados LILACS, que foi adaptada para as demais bases analisadas.

Quadro 2: Estratégia de busca realizada nas bases de dados. Teresina, PI, 2021.

\begin{tabular}{|l|}
\hline \multicolumn{1}{|c|}{ Estratégia de busca } \\
\hline P: (mh:(enfermeiro)) OR (papel do enfermeiro) \\
I: (mj:(classificação)) OR (enfermagem de urgência) \\
Co: (mh:(cartão de triagem)) OR (classificação de manchester) \\
\hline ((enfermeiros) OR (papel do enfermeiro)) AND ((mj:(classificação)) OR (enfermagem de urgência)) AND \\
((mh:(cartão de triagem)) OR (classificação de manchester)) \\
\hline
\end{tabular}

Fonte: Autores.

A princípio, foram localizadas 14 produções, dos quais 10 foram selecionadas para a leitura na integra. Após a leitura, foram selecionados 5 estudos que atenderam aos critérios de inclusão e exclusão, assim, sendo selecionados para o estudo. A Figura 1 demonstra o processo realizado para a identificação, seleção, elegibilidade, exclusão e a inclusão dos estudos, segundo as recomendações do Preferred Reporting Items for Systematic Reviews and Meta-Analyses (PRISMA). 
Figura 1: Fluxograma para identificação e seleção dos artigos.

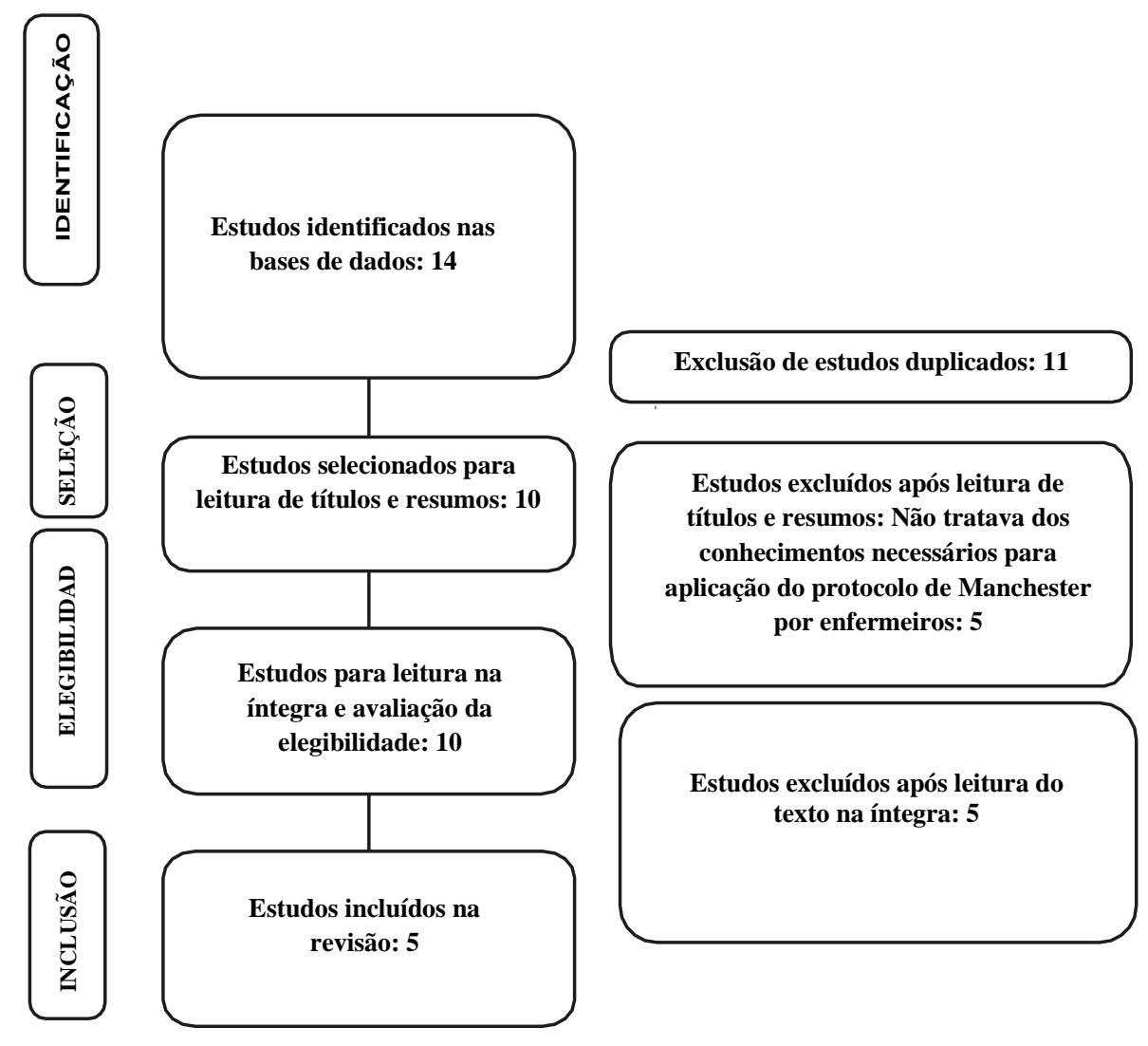

Fonte: Autores.

A análise e a síntese qualitativa dos dados foram realizadas de forma descritiva, e as produções selecionadas foram organizadas em planilhas no Microsoft Excel, procedendo-se com a construção de quadros de acordo com as variáveis identificadas. Ainda, realizaram-se o ordenamento do material e a classificação por similaridade semântica, o que possibilitou a construção de uma categoria temática.

\section{Resultados}

A coleta dos dados foi realizada com auxílio de instrumento próprio, contendo informações sobre o ano e país, delineamento da pesquisa e principais resultados. A análise e a síntese qualitativa dos dados foram realizadas de forma descritiva, e as produções selecionadas foram organizadas em quadros. 
Quadro 3: Síntese dos artigos analisados e resultados obtidos. Teresina, PI, Brasil, 2021.

\begin{tabular}{|c|c|c|}
\hline $\begin{array}{l}\text { Delineamento } \\
\text { do estudo }\end{array}$ & Ano / país & Resultados \\
\hline $\begin{array}{l}\text { Estudo } \\
\text { transversal }\end{array}$ & Brasil/ 2021 & $\begin{array}{l}\text { Destaca que para se tornar classificador, o enfermeiro deve ser treinado e acompanhado } \\
\text { periodicamente de acordo com a ferramenta de classificação, pois assim este obterá } \\
\text { habilitação para o manejo do instrumento e também para aplicação de raciocínio clínico, } \\
\text { tornando o processo menos mecanizado e mais humanizado. O conhecimento e integração } \\
\text { da classificação com parte integrante da PNH é fundamental, pois apenas o uso do PM sem } \\
\text { a PNH, torna-se insuficiente, pois juntos envolvem sinais, sintomas e aspectos culturais e } \\
\text { sociais, que podem ser um ponto fundamental para a adequada avaliação de risco de cada } \\
\text { pessoa de forma diferenciada. }\end{array}$ \\
\hline $\begin{array}{l}\text { Estudo } \\
\text { etnográfico }\end{array}$ & Brasil / 2021 & $\begin{array}{l}\text { Refere que o trabalho de classificação também depende da informação dada pelo paciente e } \\
\text { da relação que se estabelece entre o paciente e o profissional. o julgamento dos enfermeiros } \\
\text { comporta maior complexidade e diferenciação do que apenas o registro informatizado de } \\
\text { sintomas. Para além do relato do usuário, o enfermeiro utiliza a sua percepção, e, não poucas } \\
\text { vezes, surgem tensões entre a experiência do enfermeiro e a manifestação dos sinais e } \\
\text { sintomas por parte dos usuários. }\end{array}$ \\
\hline $\begin{array}{l}\text { Estudo de } \\
\text { confiabilidade }\end{array}$ & Brasil / 2018 & $\begin{array}{l}\text { Para tomar a decisão na classificação de risco, o enfermeiro integra a avaliação da queixa } \\
\text { apresentada pelo paciente com o seu conhecimento adquirido durante a formação e a vida } \\
\text { profissional, bem como o ambiente de cuidado no qual está inserido }\end{array}$ \\
\hline Estudo de caso & Brasil / 2017 & $\begin{array}{l}\text { O enfermeiro que atua na classificação de risco deve possuir algumas habilidades } \\
\text { indispensáveis para uma assistência qualificada. Dentre elas, citam-se a escuta qualificada, } \\
\text { o raciocínio clínico e a agilidade para tomada de decisões, a avaliação e o detalhamento } \\
\text { correto da queixa apresentada pelo usuário e o conhecimento da rede assistencial para } \\
\text { realizar, de forma efetiva, os encaminhamentos necessários. }\end{array}$ \\
\hline $\begin{array}{l}\text { Estudo de } \\
\text { confiabilidade }\end{array}$ & Brasil / 2016 & $\begin{array}{l}\text { A competência clínica do enfermeiro que atua na classificação de risco tendo o STM como } \\
\text { instrumento direcionador perpassa pela tomada de decisão clínica que pode ser descrita em } \\
\text { três fases: identificação do problema (queixa principal) e escolha do fluxograma de } \\
\text { apresentação, avaliação das alternativas, ou seja, verificação, de forma ordenada dos sinais } \\
\text { e sintomas apresentados pelo paciente e escolha do discriminador da classificação e, por fim, } \\
\text { indicação apropriada do nível de prioridade do paciente }\end{array}$ \\
\hline
\end{tabular}

Fonte: Autores.

\section{Discussão}

Os achados desse estudo revelam a escassez de informações acerca do tema, evidenciando a importância de serem produzidas mais obras sobre a relação entre o protocolo de Manchester e os enfermeiros, profissionais estes que, cada vez mais, vem tornando-se responsáveis por este protocolo nas urgências. Dos artigos englobados na amostra, a maioria foi produzida recentemente, no ano de 2021, o que demostra a pouca demanda em relação a um assunto de tamanha relevância.

O enfermeiro, frete ao protocolo de Manchester, possui múltiplas responsabilidades, das quais: diminuir o tempo de espera para atendimento, encaminhar rapidamente as situações mais graves, otimizar os recursos, avaliar as principais necessidades dos pacientes, classificação do atendimento de acordo com a condição clínica do cliente, ao invés de ser por ordem de chegada à triagem (Lima Lima, S \& de Paula, A. S. 2017).

O protocolo de Manchester auxilia na organização da ordem dos atendimentos, permitindo que os pacientes, ao invés de serem atendidos por ordem de chegada, sejam acolhidos e, após, possam ser atendidos conforme o nível de gravidade identificando, assim, os casos que não podem esperar atendimento devido ao risco de evolução para o óbito (Andrade, 2015).

É importante ressaltar que a avaliação deve ser sistêmica e que os dados devem ser reunidos para fornecer os problemas apresentados pelo paciente. O profissional da classificação de risco deve ter experiência suficiente em cuidados em emergência, ser capacitado profissionalmente e ter boas relações interpessoais para compreender o paciente (Jones et al., 2010).

Dentro do sistema de Manchester, a cor vermelha aponta para a necessidade de atendimento imediato (emergência), não havendo margem para espera. São exemplos de queixas que imediatamente são identificadas como classificação vermelha: Politraumatismo grave, TCE grave, Coma, Comprometimento da coluna cervical, Parada cardiorrespiratória, desconforto 
respiratório grave, Dor no peito e dispneia, Ferimentos perfurocortantes (armas de fogo), queimaduras em grande escala (Aguiar, 2019).

A cor laranja indica que o quadro foi classificado como muito urgente, e necessita de atendimento relativamente imediato, porém, não tão urgente como na cor vermelha, que apresenta um tempo limite de até 10 minutos. Situações e sintomas prontamente identificados como classificação laranja são: êmese com dor severa, lesão no abdome com histórico significativo de incidente, gravidez com histórico ou risco de convulsão (Amaral, 2017).

A cor amarela significa que o quadro é urgente, mas pode aguardar para atendimento, nesse caso o tempo de espera é de até 60 minutos. São situações prontamente identificadas como classificação amarela: enxaqueca intensa, dor torácica elevada, dor abdominal extrema, qualquer dor elevada, queda no nível de consciência, vertigem ou síncope, sangramentos, Crises de asma, febre alta (Soares Acl et al., 2018). A cor verde significa que o caso é pouco urgente e pode aguardar atendimento por até 120 minutos, ou ser encaminhado para outros serviços de saúde, em caso de superlotação, esse paciente poderá, eventualmente, ser encaminhado para a UPA de sua referência, após contato telefónico prévio ou por documento escrito, com garantia de atendimento (Aguiar, 2019). A cor azul sinaliza para uma situação não urgente e que pode esperar por atendimento, ou ser redirecionado para outros serviços de atendimento em saúde. Para a cor azul, o tempo de espera é de até 4 horas.

As primeiras noções em classificação de risco surgiram no período das guerras napoleônicas em 1800. O cirurgião Dominique Jean Larrey, general médico do exército de napoleão, em meio ao campo de batalha, criou um sistema de triagem para o atendimento de soldados feridos durante a batalha. Com a chegada dos anos 80 , a forma de triar já se adaptava ao local em que era empregada, sendo realizada por profissionais diversos como médicos e enfermeiros. O protocolo de Manchester foi criado pelo médico Kevin Mackway-Jones e sua equipe no Manchester Royal Infirmary, em 1997. Porém, Kevin iniciou as pesquisas para sua criação em 1997, quando se reuniu com enfermeiros e médicos para formalizar um padrão no atendimento de urgência. O foco do grupo era desenvolver alguns parâmetros como uma nomenclatura universal, procedimentos e métodos de triagem comuns a todos, criar um guia de auditoria e um programa de capacitação para a triagem. Logo em seguida, o protocolo se difundiu pelo reino unido e, por conseguinte, pelo resto do mundo. No ano de 2000, esse Sistema entrou em uso em alguns hospitais portugueses (Amaral, 2017).

No Brasil, o primeiro estado a adotar esse protocolo foi Minas gerais, em 2010, através de um acordo realizado entre o estado de minas gerais e o grupo português de triagem, que possuíam os direitos de tradução e uso do protocolo para a língua portuguesa. Um fator de suma importância para a viabilização desse acordo foi a criação do grupo brasileiro de classificação de risco (GBCR), responsável por promover auditorias e revisões, que atualmente detém autorização para regular o protocolo no país. Desde os anos 2000, diversas instituições de saúde de diferentes níveis populacionais e realidades econômicas vêm implementando esse sistema, como exemplo as localizadas em países como Alemanha, Áustria, Portugal, México e Espanha (Vieira et al., 2017).

$\mathrm{O}$ atendimento de urgência necessita cada vez mais de profissionais capacitados para suprir o aumento na demanda, decorrente de acidentes, violência e outras enfermidades. Na equipe de enfermagem, o enfermeiro tem papel privativo na realização da classificação de risco. Apesar de o ministério da saúde afirmar que essa classificação possa ser realizada por qualquer profissional da saúde de nível superior, o enfermeiro é designado como o profissional mais adequado a essa função. Essa competência legal do enfermeiro para tomar frente à classificação é respaldada por resolução do COFEN (Oliveira \& Viana, 2018).

De acordo com a resolução COFEN 423/2012 dispõe que o enfermeiro deve apresentar conhecimentos, habilidades e competências que assegurem o rigor técnico cientifico ao procedimento. O Código de Ética dos Profissionais de Enfermagem (CEPE), aprovado pela Resolução Cofen 311/2007, artigo 13, seção I, discorre sobre responsabilidades e deveres dos profissionais de enfermagem, que diz: “Avaliar criteriosamente sua competência técnica, científica, ética e legal e somente aceitar 
encargos ou atribuições, quando capaz de desempenho seguro para si e para outrem". Essa resolução respalda o enfermeiro realizar a capacitação de risco, compreendendo todas as etapas da enfermagem como exame físico, Sae e a supervisão da equipe de enfermagem (Vieira et al., 2017).

$\mathrm{O}$ atendimento de urgência hospitalar consiste em uma área que exige um amplo conhecimento por parte do enfermeiro acerca das diversas situações de saúde e este deve deter o controle sobre as peculiaridades da assistência, como a resolução rápida de problemas, a destreza e o rápido tirocínio tendo em vista a condição do paciente e a escassez de tempo para tomada de decisão. O profissional deve intervir de maneira qualificada, o olhar clinico sobre as queixas irão direcionar para uma linha de raciocínio que determinará o risco, isso ocorre por meio da análise e entrevista, aferição dos sinais vitais, realização do exame físico e exames complementares, que definiram a necessidade de cada caso. Esse profissional é responsável por garantir o atendimento imediato ao paciente mais grave, além de organizar os demais casos de menor gravidade, garantindo a continuidade e a eficiência do serviço de urgência, que é indispensável para dar seguimento às próximas etapas do atendimento (Oliveira \& Viana, 2018).

O conhecimento teórico é enfatizado como essencial para a realização da classificação de risco. O profissional de enfermagem necessita estar amplamente qualificado nas situações cirúrgicas, psicossociais e clinicas dos pacientes, levando em consideração a ampla variedade de problemas que estão atrelados aos serviços de urgência e emergência. $O$ enfermeiro deve apresentar uma percepção do perfil epidemiológico da população que busca o serviço de urgência, assim como a fisiopatologia das alterações mais recorrentes para se determinar um padrão de prioridades. A classificação de risco baseado no protocolo de Manchester é uma ação que deve ser realizada pelo enfermeiro, com preferência ao que possui conhecimento em urgência e após uma capacitação especifica para realização desse serviço (Soares Acl et al.,2018).

\section{Conclusão}

Além de concluir sobre a necessidade de maiores produções acerca do assunto, o estudo aponta $\mathrm{O}$ enfermeiro da triagem de urgência deve ser ágil, capaz de avaliar, orientar e tratar o paciente, baseado em seus conhecimentos fisiopatológicos, tecnológicos e de tratamentos. Deve apresentar esculta qualificada, garantindo intervenções seguras ao paciente. Trabalhando em equipe, o enfermeiro deve ser rápido e preciso na tomada de decisão e encaminhamento ao médico. A avaliação do profissional enfermeiro deve discriminar, de forma clara, qual o encaminhamento necessário ao paciente após a classificação ser recebida.

\section{Referências}

Aguiar, B. R. D. S. (2019). A importância da implantação do protocolo de Manchester nas unidades de pronto atendimento: uma revisão bibliográfica. Trabalho de Conclusão de Curso (Graduação em Enfermagem) - Faculdade de Ciências da Educação e Saúde, Centro Universitário de Brasília

Aragão Azevêdo Viana, S. (n.d.). Atribuições Do Enfermeiro Na Classificação De Risco Em Unidades De Urgência E Emergência: uma pesquisa bibliográfica. IESP agora é UNIESP | Cursos de Graduação e Pós-Graduação.

Araújo Dos Reis, E. (2018). Desafios Dos Enfermeiros Na Aplicação Do Protocolo De Manchester: Uma Revisão Da Literatura [Dissertação de conclusão de curso]. Centro Universitário de Brasília.

Bonzi, A. R. B. (2018). Enfermagem e protocolo de Manchester: revisão bibliográfica. In: Anais III CONBRACIS. ISSN: 2525-6696“.

Carapinheiro, G., Chioro, A., Andreazza, R., Spedo, S. M., Souza, A. L. M. D., Araújo, E. C. D., \& Cecilio, L. C. D. O. (2021). Os enfermeiros e o Manchester: reconfiguração do processo de trabalho e do cuidado em emergência? Revista Brasileira de Enfermagem, 74.

Cunha vieira, d. et al (2017). As Principais Dificuldades Enfrentadas Pelo Enfermeiro No Serviço De Urgência E Emergência, Utilizando O Protocolo De Manchester. centro universitário de desenvolvimento do centro oeste.

Duarte, A. F. V. (2017). O papel do enfermeiro no acolhimento com classificação de risco nos serviços de urgência e emergência: revisão integrativa. 2017. 48f. Trabalho de Conclusão de Curso (Bacharelado em Enfermagem) - Centro de Formação de Professores, Universidade Federal de Campina Grande, Cajazeiras, Paraíba, Brasil.

Freitas do amaral, S. (2017). O Uso Do Protocolo De Manchester Pode Auxiliar No Atendimento Humanizado Em Uma Emergência? [Tese de conclusão de curso, Instituto Federal De Educação, Ciência E Tecnologia Do Rio Grande Do Sul - Câmpus Porto Alegre]. 
Research, Society and Development, v. 10, n. 14, e47101421635, 2021

(CC BY 4.0) | ISSN 2525-3409 | DOI: http://dx.doi.org/10.33448/rsd-v10i14.21635

https://www.iesp.edu.br/sistema/uploads/arquivos/publicacoes/atribuicoes-do-enfermeiro-na-classificacao-de-risco-em-unidades-de-urgencia-e-emergenciauma-pesquisa-bibliografica.pdf

Ibiapina, G. R. et al (2014). Urgências e emergências clinicas em pronto-socorro consulta rápida. ideia.

Lima Lima, S., \& de Paula, A. S. (2017). Atuação do enfermeiro na aplicação do Protocolo de Manchester em unidades de urgência e emergência. ANAIS SIMPAC, $8(1)$.

Mendes, K. D. S., Silveira, R. C. D. C. P., \& Galvão, C. M. (2019). Uso de gerenciador de referências bibliográficas na seleção dos estudos primários em revisão integrativa. Texto \& Contexto-Enfermagem, 28.

Nunes, B. X., Câmara, D. V., Renovato, L., Montefusco, S. R. A., \& Amaral, M. S. (2018). Atuação Do Enfermeiro No Acolhimento Com Classificação De Risco: Caracterização Do Atendimento Mediante Protocolos: Uma Revisão Da Literatura Nurse's Activities In Hospital With Risk Classification: Characterization Of Attendance Through Protocols: A REVIEW.

Oliveira, F. (2017). Adaptação cultural e validação de conteúdo do sistema de triagem de Manchester versão inglesa para o português do Brasil. [Dissertação de doutorado]. Universidade Federal de Minas Gerais.

Querfeld, U., \& Schaefer, F. (2020). Cardiovascular risk factors in children on dialysis: an update. Pediatric Nephrology, 35(1), 41-57.

Rates, H. F., Cavalcante, R. B., Alves, M., Santos, R. C., Machado, R. M., \& Macêdo, A. S. (2018). O (in) visível no cotidiano de trabalho de enfermeiros no acolhimento com classificação de risco. Revista Eletrônica de Enfermagem, 20.

Roncalli, A. A., de Oliveira, D. N., Silva, I. C. M., Brito, R. F., \& da Fonseca Viegas, S. M. (2017). Protocolo de manchester e população usuária na classificação de risco: visão do enfermeiro. Revista Baiana de Enfermagem 31 ,(2). ,

Sacoman, T. M., Beltrammi, D. G. M., Andrezza, R., Cecílio, L. C. D. O., \& Reis, A. A. C. D. (2019). Implantação do Sistema de Classificação de Risco Manchester em uma rede municipal de urgência. Saúde em Debate, 43, 354-367.

Soares, A. C. L., Brasileiro, M., \& de Souza, D. G. (2018). Acolhimento com classificação de risco: atuação do enfermeiro na urgência e emergência. Revista Recien-Revista Científica de Enfermagem, 8(22), 22-33.

Sousa, L. M. M., Marques-Vieira, C. M. A., Severino, S. S. P., \& Antunes, A. V. (2017). A metodologia de revisão integrativa da literatura em enfermagem. $N^{o} 21$ Série 2-Novembro 2017, 17.

Souza, C. C. D., Araújo, F. A., \& Chianca, T. C. M. (2015). Produção científica sobre a validade e confiabilidade do Protocolo de Manchester: revisão integrativa da literatura. Revista da Escola de Enfermagem da USP, 49, 144-151. 